A.G. Ramm, Global convergence for ill-posed equations with monotone operators: the dynamical systems method, J.Phys A, 36, (2003), L249-L254 


\title{
Global convergence for ill-posed equations with monotone operators: the dynamical systems method *
}

\author{
A.G. Ramm \\ Mathematics Department, Kansas State University, \\ Manhattan, KS 66506-2602, USA \\ Email: ramm@math.ksu.edu
}

Consider an operator equation $F(u)=0$ in a real Hilbert space. Let us call this equation ill-posed if the operator $F^{\prime}(u)$ is not boundedly invertible, and well-posed otherwise. If $F$ is monotone $C_{\text {loc }}^{2}(H)$ operator, then we construct a Cauchy problem, which has the following properties: 1) it has a global solution for an arbitrary initial data, 2) this solution tends to a limit as time tends to infinity, 3) the limit is the minimum norm solution to the equation $F(u)=0$.

Example of applications to linear ill-posed operator equation is given.

\section{Introduction}

Many physical problems can be formulated as operator equations. In this paper a general convergence theorem is proved for solving operator equations with monotone operators. Consider an operator equation

$$
F(u):=\mathcal{B}(u)-f=0, \quad f \in H,
$$

where $\mathcal{B}$ is a monotone, nonlinear, $C_{\text {loc }}^{2}$ operator in a real Hilbert space $H$, i.e, $\sup _{u \in B\left(u_{0}, R\right)}\left\|F^{(j)}(u)\right\| \leq M_{j}(R):=M_{j}, j=0,1,2$, where $R>0$ is arbitrary, $B\left(u_{0}, R\right):=\left\{u:\left\|u-u_{0}\right\| \leq R\right\}, F^{(j)}(u)$ is the Fréchet derivative. Let

*Math subject classification: 34R30, 35R25, 35R30, 37C35, 37L05, 37N30, 47A52, 47J06, 65M30, 65N21 
$N:=\{z: F(z)=0\}$. It is known that $N$ is convex and closed under our assumptions. Assume that $N$ is not empty. Then it contains the unique minimum norm element $y: F(y)=0, \quad\|y\| \leq\|z\|, \forall z \in N$. These assumptions hold throughout and are not repeated.

Let $\dot{u}$ denote derivative with respect to time. Consider the dynamical system ( the Cauchy problem ):

$$
\dot{u}=\Phi(t, u), u(0)=u_{0} ; \Phi:=-A_{\epsilon}^{-1}[F+\epsilon u],
$$

where $A_{\epsilon}:=A+\epsilon I, A:=F^{\prime}(u), I$ is the identity operator, and $\epsilon=\epsilon(t)>0$ is a continuously differentiable, monotone, decaying to zero as $t \rightarrow \infty$, function on $[0, \infty)$. Specifically, we will use $\epsilon=c_{1}\left(c_{0}+t\right)^{-b}$, where $c_{1}, c_{0}$ and $b$ are positive constants, $b \in(0,1)$, and assume throughout (without repeating), that $|\dot{\epsilon}| \epsilon^{-1} \leq 0.25$. Note that $\Phi(t, u)$ is locally Lipschitz with respect to $u \in H$ and continuous with respect to $t \geq 0$ under our assumptions. Thus problem (1.2) has a unique local solution. We want to solve equation (1.1) by solving (1.2), and proving that for any initial $u_{0}$ the following three results hold:

$$
\exists u(t) \forall t>0 ; \quad \exists u(\infty):=\lim _{t \rightarrow \infty} u(t) ; \quad F(u(\infty))=0 .
$$

Moreover, we prove that the solution $u \in B\left(u_{0}, R\right) \forall t \geq 0$, where $R:=3 r$, and $r:=\|y\|+\left\|u_{0}\right\|$.

Problem (1.1) with noisy data $f_{\delta},\left\|f_{\delta}-f\right\| \leq \delta$, given in place of $f$, generates the problem:

$$
\dot{u}_{\delta}=\Phi_{\delta}\left(t, u_{\delta}\right), u_{\delta}(0)=u_{0},
$$

The solution $u_{\delta}$ to (1.4), calculated at a suitable stopping time $t=t_{\delta}$, converges to $y$ :

$$
\lim _{\delta \rightarrow 0}\left\|u_{\delta}\left(t_{\delta}\right)-y\right\|=0 .
$$

The choice of $t_{\delta}$ with this property is called the stopping rule. One has usually $\lim _{\delta \rightarrow 0} t_{\delta}=\infty$.

We do not restrict the growth of nonlinearity at infinity and do not assume that the initial approximation $u_{0}$ is close to the solution $y$ in any sense. Usually (e.g., see [2]) convergence theorems for Newton-type methods for solving nonlinear equation (1.1) have the assumption that the initial data $u_{0}$ is close to $y$. We obtain a global convergence result for a continuous regularized Newton-type method (1.2). This result is stated in Theorem 1, and proved in Section 2. 
Theorem 1. For any choice of $u_{0}$ problem (1.2) has a global solution, this solution stays in the ball $B\left(u_{0}, R\right)$, and (1.3) holds. If $u_{\delta}(t)$ solves (1.4), then there is a $t_{\delta}$ such that $\lim _{\delta \rightarrow 0}\left\|u_{\delta}\left(t_{\delta}\right)-y\right\|=0$.

The proof uses essentially the following result which is obtained in [1].

Theorem 2. Let $\gamma(t), \sigma(t), \beta(t) \in C\left[t_{0}, \infty\right)$ for some real number $t_{0}$. If there exists a positive function $\mu(t) \in C^{1}\left[t_{0}, \infty\right)$ such that

$$
0 \leq \sigma(t) \leq \frac{\mu(t)}{2}\left[\gamma(t)-\frac{\dot{\mu}(t)}{\mu(t)}\right], \quad \beta(t) \leq \frac{1}{2 \mu(t)}\left[\gamma(t)-\frac{\dot{\mu}(t)}{\mu(t)}\right], \quad g_{0} \mu\left(t_{0}\right)<1
$$

where $g_{0}$ is the initial condition in (1.7), then a nonnegative solution $g$ to the following differential inequality:

$$
\dot{g}(t) \leq-\gamma(t) g(t)+\sigma(t) g^{2}(t)+\beta(t), \quad g\left(t_{0}\right)=g_{0},
$$

satisfies the estimate:

$$
0 \leq g(t) \leq \frac{1-\nu(t)}{\mu(t)}<\frac{1}{\mu(t)}
$$

for all $t \in\left[t_{0}, \infty\right)$, where

$$
0<\nu(t)=\left(\frac{1}{1-\mu\left(t_{0}\right) g\left(t_{0}\right)}+\frac{1}{2} \int_{t_{0}}^{t}\left(\gamma(s)-\frac{\dot{\mu}(s)}{\mu(s)}\right) d s\right)^{-1} .
$$

There are several novel features in this result. First, differential equation, which one gets from (1.7) by replacing the inequality sign by the equality sign, is a Riccati equation, whose solution may blow up in a finite time, in general. Conditions (1.6) guarantee the global existence of the solution to this Riccati equation with the initial condition (1.7). Secondly, this Riccati differential equation cannot be integrated analytically by separation of variables, in general. Thirdly, the coefficient $\sigma(t)$ may grow to infinity as $t \rightarrow \infty$, so that the quadratic term does not necessarily has a small coefficient, or the coefficient smaller than $\gamma(t)$. Without loss of generality one may assume $\beta(t) \geq 0$ in Theorem 2. In [4] and [5] one finds a description and applications of DSM (dynamical systems method) and some remarks about discrepancy principle, which are useful in treating problems with noisy data. Many physical problems can be formulated as operator equations with monotone operators. We mention the theory of passive networks (see [7] and [8], Chapter 3) as just one of many examples. 


\section{Proof of Theorem 1}

Let us sketch the proof. Denote $w:=u-V,\|w\|:=g, v:=\|V-y\|$. Clearly $\|u(t)-y\| \leq g+v$. We will prove that $\lim _{t \rightarrow \infty} g=\lim _{t \rightarrow \infty} v=0$.

Let $V$ solve the equation

$$
F(V)+\epsilon(t) V=0
$$

Under our assumptions on $F$, it is known that (2.1) has a unique solution for every $t>0$, and $\lim _{t \rightarrow \infty}\|V(t)-y\|=0$. One can prove that $\sup _{t \geq 0}\|V\| \leq$ $\|y\|, V$ is differentiable, and $\|\dot{V}(t)\| \leq\|y\| \dot{\epsilon}(t) \mid / \epsilon(t)$. We will show that the global solution $u$ to (1.2) does exist, and $\lim _{t \rightarrow \infty}\|u(t)-V(t)\|=0$. This is done by deriving a differential inequality for $w$, and by applying Theorem 2 to $g=\|w\|$. Since $\|u(t)-y\| \leq g+v$, it then follows that (1.3) holds. We also check that $u(t) \in B\left(u_{0}, R\right)$, where $R:=3\left(\|y\|+\left\|u_{0}\right\|\right)$, for any choice of $u_{0}$ and a suitable choice of $\epsilon=\epsilon(t)$.

Let us derive the differential inequality for $w$. One has

$$
\dot{w}=-\dot{V}-A_{\epsilon(t)}^{-1}(u)[F(u(t))-F(V(t))+\epsilon(t) w],
$$

and $F(u)-F(V)=A w+K$, where $\|K\| \leq M_{2} g^{2} / 2, g:=\|w\|$. Multiply (2.2) by $w$, use monotonicity of $F$, i.e., the property $A \geq 0$, and the estimate $\|\dot{V}\| \leq\|y\||| \dot{\epsilon} \mid / \epsilon$, and get:

$$
\dot{g} \leq-g+\frac{0.5 M g^{2}}{\epsilon}+\|y\| \frac{|\dot{\epsilon}|}{\epsilon}
$$

where $M:=M_{2}$. Inequality (2.3) is of the type (1.7): $\gamma=1, \sigma=0.5 M / \epsilon$, $\beta=\|y\| \frac{|\dot{\epsilon}|}{\epsilon}$. Choose $\mu(t)=2 M / \epsilon(t)$. Clearly $\mu \rightarrow \infty$ as $t \rightarrow \infty$. Let us check three conditions (1.6). One has $\frac{\dot{\mu}(t)}{\mu(t)}=|\dot{\epsilon}| / \epsilon$. Take $\epsilon=c_{1}\left(c_{0}+t\right)^{-b}$, where $c_{j}>0$ are constants, $0<b<1$, and choose these constants so that $|\dot{\epsilon}| / \epsilon<1 / 2$, e.g., $\frac{b}{c_{0}}=\frac{1}{4}$. Then the first condition (1.6) is satisfied. The second condition (1.6) holds if $\left(^{*}\right) 8 M\|y\||\| \dot{\epsilon}| \epsilon^{-2} \leq 1$. One has $\epsilon(0)=c_{1} c_{0}^{-b}$. Choose $\epsilon(0)=4 M r$. Then $|\dot{\epsilon}| \epsilon^{-2}=b c_{1}^{-1}\left(c_{0}+t\right)^{b-1} \leq b c_{0}^{-1} c_{1}^{-1} c_{0}^{b}=\frac{1}{4 \epsilon(0)}=$ $\frac{1}{16 M r}$. Thus, the second condition (1.6) holds. The last condition (1.6) holds because $2 M\left\|u_{0}-V_{0}\right\| / \epsilon(0) \leq \frac{2 M r}{4 M r}=\frac{1}{2}<1$.

Thus, by Theorem 2, $g=\|w(t)\|<\frac{\epsilon(t)}{2 M} \rightarrow 0$ when $t \rightarrow \infty$, and $\| u(t)-$ $u_{0}\|\leq g+\| V-u_{0} \| \leq g(0)+r \leq 3 r$. This estimate implies the global existence of the solution to (1.2), because if $u(t)$ would have a finite maximal interval 
of existence, $[0, T)$, then $u(t)$ could not stay bounded when $t \rightarrow T$, and this contradicts the boundedness of $\|u(t)\|$, which follows from our estimates: $\|u(t)\| \leq 4 r$. We have proved the first part of Theorem 4.2, namely properties (1.3).

To derive a stopping rule we argue as follows. One has $\left\|u_{\delta}(t)-y\right\| \leq$ $\left\|u_{\delta}(t)-V(t)\right\|+\|V(t)-y\|:=g_{\delta}+v$. We have proved that $\lim _{t \rightarrow \infty} v(t):=0$. The rate of decay of $v(t)$ can be arbitrarily slow, in general. Additional assumptions, e.g., the source-type ones, can be used to estimate the rate of decay of $v(t)$. One can derive differential inequality similar to (2.3) for $g_{\delta}:=\left\|u_{\delta}(t)-V(t)\right\|$, and estimate $g_{\delta}$ using (1.8). The analog of (2.3) for $g_{\delta}$ contains additional term $\delta / \epsilon$ on the right-hand side. If $16 M \delta \leq \epsilon^{2}$, then conditions (1.6) hold, and $g_{\delta}<\frac{\epsilon(t)}{2 M}$. Let $t_{\delta}$ be the root of the equation $\epsilon^{2}(t)=16 M \delta$. Then $\lim _{\delta \rightarrow 0} t_{\delta}=\infty$, and $\lim _{\delta \rightarrow 0}\left\|u_{\delta}\left(t_{\delta}\right)-y\right\|=0$, because $\left\|u_{\delta}\left(t_{\delta}\right)-y\right\| \leq v\left(t_{\delta}\right)+g_{\delta}, \lim _{t_{\delta} \rightarrow \infty} g_{\delta}\left(t_{\delta}\right)=0$ and $\lim _{t_{\delta} \rightarrow \infty} v\left(t_{\delta}\right)=0$, but the convergence can be slow. See also [3] for the rate of convergence under source assumptions. If the rate of decay of $v(t)$ is known, then one can choose $t_{\delta}$ as the minimizer of the problem, similar to (3.13), $v(t)+g_{\delta}(t)=$ min, where the minimum is taken over $t>0$ for a fixed small $\delta>0$. This would yield a quasioptimal stopping rule. Theorem 1 is proved.

\section{Example}

Let us give an example of applications of Theorem 1. Consider a linear operator equation:

$$
A u=f .
$$

Let us denote by A) the folowing assumption:

Assumption (A): A is a linear, bounded operator in $H$, defined on all of $H$, the range $R(A)$ is not closed, so (3.1) is an ill-posed problem, there is a $y$ such that $A y=f, y \perp N$, where $N$ is the null-space of $A$.

Let $\mathcal{B}:=A^{*} A, q:=\mathcal{B} y=A^{*} f, A^{*}$ is the adjoint of $A$. Every solution to (3.1) solves

$$
\mathcal{B} u=q,
$$

and, if $f=A y$, then every solution to (3.2) solves (3.1). Choose a continuous function $\epsilon(t)>0$, monotonically decaying to zero on $\mathbb{R}_{+}$, as in Theorem 1. If $\mathcal{B}$ is a linear operator, and $F(u):=\mathcal{B} u-q$, then $F^{\prime}(u)=\mathcal{B}$, and $\Phi:=-(\mathcal{B}+\epsilon)^{-1}[\mathcal{B} u-q+\epsilon u]=-u+(\mathcal{B}+\epsilon(t))^{-1} q$. Therefore equation $(1.2)$ 
takes the form:

$$
\dot{u}=-u+(\mathcal{B}+\epsilon(t))^{-1} q, \quad u(0)=u_{0} .
$$

The operator $\mathcal{B}:=A^{*} A \geq 0$ is linear, monotone, and Theorem 1 is applicable. Therefore conclusions (1.3) hold for the solution to (3.3), and, since equations (3.1) and (3.2) are equivalent if (3.1) is solvable, one concludes that $u(\infty)=$ $y$, where $y$ is the unique minimal-norm solution to equation (3.1). Moreover, if the data are noisy, so that $f_{\delta}$ is given in place of $f$, and $\left\|f-f_{\delta}\right\| \leq \delta$, then Theorem 1 yields a stable solution to the ill-posed problem (3.1). Thus, Theorem 1 yields a method for solving arbitrary linear ill-posed problems with bounded linear operator $A$. This method works well numerically.

\section{Appendix}

For convenience of the reader and for completeness of the presentation we include a proof of Theorem 2 which is borrowed from [1].

We start with the well-known lemma (see, e.g. [6]):

Lemma. Let $f(t, w), g(t, u)$ be continuous on region $[0, T) \times D(D \subset R$, $T \leq \infty)$ and $f(t, w) \leq g(t, u)$ if $w \leq u, t \in(0, T), w, u \in D$. Assume that $g(t, u)$ is such that the Cauchy problem

$$
\dot{u}=g(t, u), \quad u(0)=u_{0}, \quad u_{0} \in D
$$

has a unique solution. If

$$
\dot{w} \leq f(t, w), \quad w(0)=w_{0} \leq u_{0}, \quad w_{0} \in D,
$$

then $u(t) \geq w(t)$ for all $t$ for which $u(t)$ and $w(t)$ are defined.

Let us now prove Theorem 2.

Proof of Theorem 2.

Let $g$ be the function from (1.7). Define the new function $w$ by the formula:

$$
w(t):=g(t) e^{\int_{t_{0}}^{t} \gamma(s) d s} .
$$

Then

$$
\dot{w}(t) \leq a(t) w^{2}(t)+b(t), \quad w\left(t_{0}\right)=g\left(t_{0}\right),
$$

where

$$
a(t)=\sigma(t) e^{-\int_{t_{0}}^{t} \gamma(s) d s}, \quad b(t)=\beta(t) e^{\int_{t_{0}}^{t} \gamma(s) d s}
$$


Consider the equation:

$$
\dot{u}(t)=\frac{\dot{f}(t)}{G(t)} u^{2}(t)-\frac{\dot{G}(t)}{f(t)} .
$$

One can check by a direct calculation that the the solution to this equation is given by the following formula (see [6]):

$$
u(t)=-\frac{G(t)}{f(t)}+\left[f^{2}(t)\left(C-\int_{t_{0}}^{t} \frac{\dot{f}(s)}{G(s) f^{2}(s)} d s\right)\right]^{-1},
$$

where $C$ is a constant. If $u(0)=u_{0}$, then $C=\frac{1}{u_{0} f^{2}(0)+G(0) f(0)}$. Define $f$ and $G$ as follows:

$$
f(t):=\mu^{\frac{1}{2}}(t) e^{-\frac{1}{2} \int_{t_{0}}^{t} \gamma(s) d s}, \quad G(t):=-\mu^{-\frac{1}{2}}(t) e^{\frac{1}{2} \int_{t_{0}}^{t} \gamma(s) d s},
$$

and consider the Cauchy problem for equation (4.1) with the initial condition $u\left(t_{0}\right)=g\left(t_{0}\right)$. Then $C$ in $(4.2)$ can be calculated:

$$
C=\frac{1}{\mu\left(t_{0}\right) g\left(t_{0}\right)-1} .
$$

From (1.6) one gets

$$
a(t) \leq \frac{\dot{f}(t)}{G(t)}, \quad b(t) \leq-\frac{\dot{G}(t)}{f(t)} .
$$

Since $f G=-1$ one has:

$$
\int_{t_{0}}^{t} \frac{\dot{f}(s)}{G(s) f^{2}(s)} d s=-\int_{t_{0}}^{t} \frac{\dot{f}(s)}{f(s)} d s=\frac{1}{2} \int_{t_{0}}^{t}\left(\gamma(s)-\frac{\dot{\mu}(s)}{\mu(s)}\right) d s .
$$

Thus

$$
u(t)=\frac{e^{\int_{t_{0}}^{t} \gamma(s) d s}}{\mu(t)}\left[1-\left(\frac{1}{1-\mu\left(t_{0}\right) g\left(t_{0}\right)}+\frac{1}{2} \int_{t_{0}}^{t}\left(\gamma(s)-\frac{\dot{\mu}(s)}{\mu(s)}\right) d s\right)^{-1}\right] .
$$

It follows from conditions (1.6) and from the second inequality in (1.6) that the solution to problem (4.1) exists for all $t \in[0, \infty)$ and the following inequality holds with $\nu(t)$ defined by $(1.9)$ :

$$
1>1-\nu(t) \geq \mu\left(t_{0}\right) g\left(t_{0}\right) .
$$


From Lemma and from formula (4.3) one gets:

$$
g(t) e^{\int_{t_{0}}^{t} \gamma(s) d s}:=w(t) \leq u(t)=\frac{1-\nu(t)}{\mu(t)} e^{\int_{t_{0}}^{t} \gamma(s) d s}<\frac{1}{\mu(t)} e^{\int_{t_{0}}^{t} \gamma(s) d s},
$$

and thus estimate (1.8) is proved. Theorem 2 is proved.

\section{References}

[1] Airapetyan R., Ramm A.G., Dynamical systems and discrete methods for solving nonlinear ill-posed problems, Appl.Math.Reviews, vol. 1, Ed. G. Anastassiou, World Sci. Publishers, Singapore, (2000), pp.491-536.

[2] Deimling K., Nonlinear functional analysis, Springer Verlag, Berlin, 1985.

[3] Kaltenbacher B., Neubauer A., Ramm A.G., Convergence rates of the continuous regularized Gauss-Newton method, Jour. Inv. Ill-Posed Probl., 10, N3, (2002), 261-280.

[4] Ramm A.G., Dynamical systems method for solving operator equations, Communic. in Nonlinear Sci. and Numer. Simulation, 9, N2, (2003)

[5] Ramm A.G., Discrepancy principle for the dynamical systems method, Communic. in Nonlinear Sci. and Numer. Simulation, 9, N, (2003)

[6] Kamke E., Differentialgleichungen. Lösungmethoden und Lösungen, Chelsea, New York, 1974.

[7] Ramm A.G., Stationary regimes in passive nonlinear networks, in "Nonlinear Electromagnetics", Ed. P.L.E. Uslenghi, Acad. Press, N. Y., 1980, pp. 263-302.

[8] Ramm A.G., Theory and applications of some new classes of integral equations, Springer-Verlag, New York, 1980 